Original Paper http://ajol.info/index.php/ijbcs http://indexmedicus.afro.who.int

\title{
Diversité et vertus thérapeutiques des ressources floristiques de production de miel au Bénin
}

\author{
Sênan Christa LOKOSSOU ${ }^{1,2}$, Fidèle Paul TCHOBO ${ }^{1 *}$, Andriano Jospin DJOSSOU ${ }^{1}$, \\ Hounnankpon YEDOMONHAN ${ }^{2}$ et Akpovi AKOEGNINOU ${ }^{2}$ \\ ${ }^{1}$ Unité de Recherche en Génie Enzymatique et Alimentaire, Université d'Abomey-Calavi, 01 BP 2009 Cotonou, \\ Bénin. \\ ${ }^{2}$ Laboratoire de Botanique et d'Ecologie végétale, Université d'Abomey-Calavi, Bénin. \\ *Auteur correspondant ; E-mail : fideletchobo@gmail.com ; Tel : (+229) 97686201
}

\section{RESUME}

L'apiculture est en plein essor au Bénin et y est pratiquée le plus souvent par les ruraux dans le but d'accroître les revenus. Cette étude visait à documenter les espèces mellifères et leurs usages en médecine traditionnelle au Bénin. L'étude a été menée en zones soudanienne, soudano-guinéenne et guinéenne. Les données ont été collectées, par le biais d'interviews, dans 24 villages à caractère apicole dans les communes de Tanguiéta, de Kandi, de Bassila, de N'dali et de Zogbodomey. Les données ont ensuite été traitées avec des outils statistiques appropriés. Trente-une espèces mellifères inductrices d'accumulation de miel appartenant à 18 familles ont été recensées. Les plus citées sont Vitellaria paradoxa, Anacardium occidentale, Parkia biglobosa, Citrus sinensis et Khaya senegalensis. Vingt-deux espèces mellifères sont utilisées à des fins médicales et les principales sollicitées sont $V$. paradoxa $(28,6 \%)$ et Mangifera indica $(12,8 \%)$. Ces 22 espèces interviennent dans le traitement de 39 pathologies dont la plus citée est le paludisme $(18,8 \%)$. Une utilisation majoritaire des écorces a été notée avec la décoction $(61,7 \%)$ comme principale forme pharmaceutique. Cette étude a permis d'apprécier le degré de connaissance des apiculteurs en termes de ressources floristiques mellifères et de leur utilisation en médecine traditionnelle.

(C) 2019 International Formulae Group. All rights reserved.

Mots clés : Espèces mellifères, médecine traditionnelle, apiculture, zone phytogéographique.

\section{Diversity and medicinal uses of melliferous species in Benin}

\begin{abstract}
Beekeeping is booming in Benin and is practiced most often by rural people in order to increase incomes. This study aimed to document the honey species and their uses in traditional medicine in Benin. The study was conducted in the Sudanese, Sudano-Guinean and Guinean zones. The data were collected through interviews in 24 beekeeping villages in Tanguieta, Kandi, Bassila, N'dali and Zogbodomey communes. The data was then processed with appropriate statistical tools. Thirty-one species inducing honey accumulation belonging to 18 families have been identified. The most cited are Vitellaria paradoxa, Anacardium
\end{abstract}


occidentale, Parkia biglobosa, Citrus sinensis and Khaya senegalensis. Twenty-two honey species are used for medical purposes and the main demands are V. paradoxa (28.6\%) and Mangifera indica (12.8\%). These 22 species are involved in the treatment of 39 pathologies, of which the most cited is malaria (18.8\%). A majority use of bark was noted with the decoction $(61.7 \%)$ as the main pharmaceutical form. This study made it possible to assess the degree of knowledge of beekeepers in terms of melliferous plant and their use in traditional medicine.

(C) 2019 International Formulae Group. All rights reserved.

Keywords: Honey species, traditional medicine, beekeeping, phytogeographical zone.

\section{INTRODUCTION}

Dans les pays pauvres comme le Bénin, l'apiculture est une vieille pratique du système de production agricole. Elle constitue l'une des rares activités qui nécessite peu d'investissements et qui fournit des revenus substantiels, en même temps qu'elle contribue à la protection de l'environnement (Schweitzer et al., 2013; Amakpe et al., 2015). Pour Fosto et al. (2014), le meilleur moyen d'accroître la production et la productivité de l'apiculture est d'en avoir une bonne connaissance. Cette connaissance fournirait des bases pour l'évaluation objective de la productivité quantitative et qualitative des abeilles des différentes régions (Dongock et al., 2004). Hormis donc l'étude des abeilles mellifiques, l'étude des plantes mellifères apparaît d'une importance capitale. En effet, puisque les abeilles dépendent des plantes pour leur existence, le développement durable de l'apiculture dans une région donnée passe par la maîtrise des relations qui existent entre ces abeilles et les plantes (Atibita et al., 2015). Au Bénin, de nombreuses études portant sur l'inventaire de la flore mellifère par la méthode des observations directes ont été réalisées (Yédomonhan et al., 2009; Amakpè et al., 2015). Toutefois, considérant que les apiculteurs sont les premiers acteurs de la filière, et que toute intervention fructueuse nécessiterait leur contribution, il s'avère aussi nécessaire de faire le point de leurs connaissances quant aux ressources mellifères en termes de diversité floristique et d'usages en médecine traditionnelle.

Selon Dro et al. (2013), dans tous les pays en voie de développement, les plantes médicinales constituent le moyen le plus utilisé, surtout en milieu rural, pour résoudre les problèmes de santé publique. Des millions de personnes utilisent en Afrique, avant tout et parfois exclusivement, la médecine traditionnelle pour leurs soins primaires de santé, parce que cette médecine demeure plus abordable et semble efficace (Béné et al., 2016). Au regard de cette réalité et tenant compte des multiples vertus attribuées aux produits de la ruche, il est essentiel de chercher à connaître l'importance des espèces mellifères en médecine traditionnelle. Cette connaissance pourrait expliquer en aval les propriétés thérapeutiques attribuées au miel.

L'objectif de cette étude est de contribuer à la connaissance des ressources floristiques de production de miel au Bénin et d'apprécier leur importance socio-médicinale.

\section{MATERIEL ET METHODES}

\section{Milieu d'étude et collecte des données}

La présente étude a été menée en République du Bénin qui s'étend entre les parallèles $6^{\circ} 15^{\prime}$ et $12^{\circ} 25^{\prime}$ de latitude Nord et $0^{\circ} 40^{\prime}$ et $3^{\circ} 45^{\prime}$ de longitude Est. Les données ont été collectées à Bassila (Département de la Donga) et à N'dali (Département du Borgou) dans la zone soudano-guinéenne ; à Tanguiéta (Département de l'Atacora) et à Kandi (Département de l'Alibori) dans la zone soudanienne ; et à Zogbodomey (Département du Zou) dans la zone guinéenne.

L'inventaire des espèces mellifères et de leurs vertus médicinales a été réalisé dans les cinq communes (Tanguiéta, Kandi, Bassila, N'Dali et Zogbodomey) et les données ont été collectées par le biais d'interviews individuelles à l'aide de questionnaires. Les villages, considérés comme unité primaire de sondage, ont été 
choisis au sein des communes suivant la méthode non probabiliste, le choix raisonné basé sur les informations recueillies (village à forte production de miel, concentration des apiculteurs) grâce aux agents des Centres d'Action Régionaux pour le Développement Rural (CARDeR). Ainsi, les enquêtes ont été menées dans 24 villages où l'apiculture est pratiquée et est en plein essor (Figure 1). Dans chacun de ces villages, le recrutement des enquêtés a été fait suivant la méthode non probabiliste dite de réseau (boule de neige). Au total, 30 apiculteurs ont été enquêtés par commune soit 150 apiculteurs. Ces apiculteurs appartenaient à six groupes sociolinguistiques et apparentés : les Bariba $(28,5 \%)$, les Ottamari $(23,3 \%)$, les Fons $(22,6 \%)$, les Yoalokpa $(14,3 \%)$, les Yoruba $(8,3 \%)$ et les Peulh $(3,0 \%)$. Leur âge moyen était de 46,36 $\pm 10,48$ ans. Les informations recueillies auprès de ces apiculteurs ont porté sur les noms vernaculaires des plantes dont les floraisons précèdent ou coïncident avec la période de récolte de miel; les plantes visitées par les abeilles parmi celles en fleurs citées; les nutriments récoltés par les abeilles; le degré de butinage des plantes (espèces fortement butinées, moyennement butinées et faiblement butinées); les plantes butinées et dont la floraison engendre l'accumulation de miel dans les ruches.

S'agissant de l'inventaire des utilisations médicinales des espèces mellifères citées, elle a été faite dans les communes et les villages sus-sélectionnés. Les apiculteurs ont systématiquement fait partie de l'échantillon en raison de leur connaissance des plantes. Ainsi, 133 apiculteurs ont pu répondre aux questionnaires et 17 guérisseurs traditionnels ont en plus été enquêtés à raison de 30 personnes par commune. Le profil socio-culturel de ces enquêtés se présente comme suit : 88,3\% étaient de sexe masculin ; $13,6 \%$ étaient alphabétisés ; $46,7 \%$ avaient au moins le niveau primaire; et $40 \%$ n'avaient reçu aucune instruction formelle. L'âge moyen était de 46,63 $\pm 11,44$ ans et $15,8 \%$ de cet échantillon avaient plus de 60 ans. Six groupes sociolinguistiques et apparentés étaient représentés: les Bariba, les YoaLokpa, les Ottamari, les Fon, les Yoruba et les Peuhl. Les informations collectées étaient : les symptômes et les maladies traités par chacune des espèces mellifères citées, les organes utilisés, les posologies et les modes d'administration des préparations issues de ces plantes.

\section{Analyse statistique}

L'identification botanique des plantes citées a été faite à l'Herbier National du Bénin par comparaison aux échantillons de référence. La nomenclature botanique utilisée est celle de Akoègninou et al. (2006). Les données collectées à travers les différentes enquêtes sur les espèces mellifères, les utilisations thérapeutiques ont été compilées en bases de données à l'aide du logiciel Microsoft Access. Ces bases ont été analysées par la statistique descriptive (fréquences, moyennes, écart-types, tableaux croisés). Des tests de $\chi 2$ ont été faits pour apprécier les liens entre les différentes variables qualitatives, et des analyses de variance ont été réalisées pour comparer les moyennes des variables quantitatives. A cet effet, le logiciel Statistical Package for the Social Sciences, version 21, a été utilisé. Le seuil de significativité retenu est de 0,05 .

Afin d'apprécier le consensus des tradi-praticiens enquêtés, le degré de consensus (DC) sur l'utilisation d'une espèce dans le traitement d'une affection a été calculé selon la formule de Bakwaye et al. (2013): $D C=\left(n_{r}-n_{0}\right)\left(n_{r}-1\right)$, où $\mathrm{n}_{\mathrm{r}}$ est le nombre total de citations enregistrées pour l'espèce $i$ et $n_{0}$ le nombre de maladies traitées avec cette espèce. Le DC d'une espèce médicinale varie de 0 (quand le nombre de maladies traitées est égal au nombre de citations enregistrées) à 1 (quand tous les participants s'accordent sur l'usage exclusif de l'espèce pour une maladie particulière). 


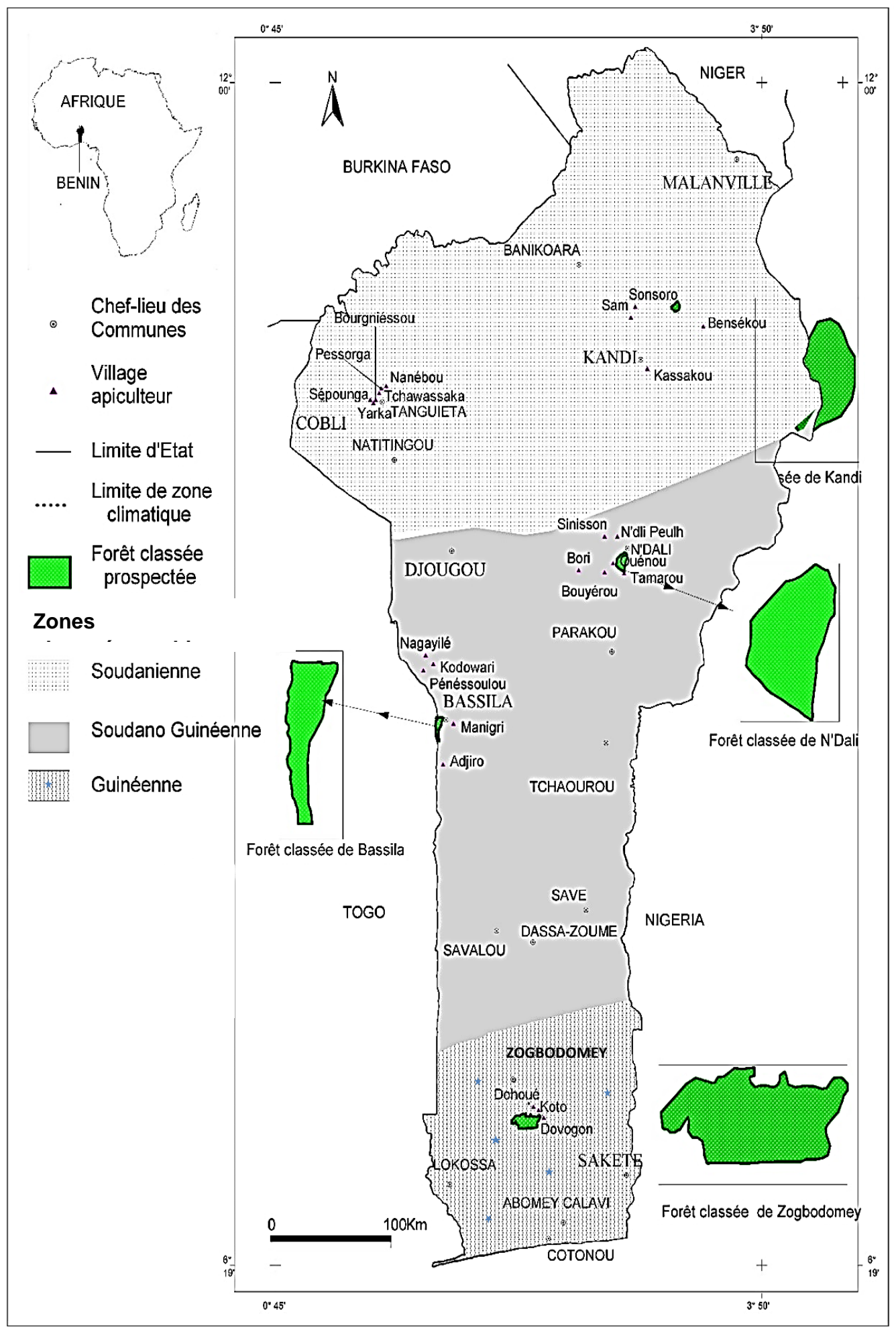

Figure 1 : Localisation géographique des 24 villages enquêtés. 


\section{RESULTATS}

\section{Ressources floristiques de production de miel selon la perception des apiculteurs}

Au total, 50 espèces végétales ont été citées par les apiculteurs comme plantes dont la floraison précède ou coïncide avec la période de récolte de miel au niveau des ruchers. Parmi elles, 45 sont visitées par les abeilles et considérées comme mellifères dont 31 espèces induisent une accumulation de miel selon les apiculteurs et sont considérées comme nectarifères.

\section{Richesse taxonomique des plantes visitées par les abeilles}

Les 45 espèces butinées appartiennent à 43 genres et 22 familles dont les plus diversifiées sont les LeguminosaeCesalpinioideae (13,33\%), les Meliaceae $(8,89 \%)$, les Leguminosae-Papilionoideae, les Leguminosae-Mimosoideae, les Anarcadiaceae, les Rubiaceae et les Rutaceae (6,66\% chacune) (Tableau 1). Les espèces les plus citées sont: $V$. paradoxa $(67,67 \%), P$. biglobosa (50,36\%), M. indica (48,87\%), A. occidentale $(32,33 \%), C$. sinensis $(20,30 \%)$ et $K$. senegalensis $(15,79 \%)$ (Figure 2). Les espèces comme Corchorus olitorius, Sorghum bicolor et Crossopteryx febrifuga ont enregistré les plus faibles fréquences de citation $(0,75 \%$ respectivement). La tendance globale de citation a varié d'une zone phytogéographique à une autre. Ainsi, $V$. paradoxa (75-88,37\%), P. biglobosa (45$72,09 \%)$ et $A$. occidentale $(18,60-70 \%)$ sont les espèces les plus citées en zones soudanienne et soudano-guinéenne alors que C. sinensis $(66,66 \%), \quad K . \quad$ senegalensis $(56,66 \%)$ et Eucalyptus camadulensis $(26,66 \%)$ ont les plus fortes fréquences de citation en zone guinéenne.

Sur la base de l'intensité de butinage, ces 45 espèces ont été réparties en trois grandes catégories : les intensément butinées, les moyennement butinées et les faiblement butinées (Tableau 1). La catégorie des taxa intensément butinés est riche de 10, 19 et sept (7) espèces respectivement pour les zones soudanienne, soudano-guinéenne et guinéenne. Les plus cités dans cette catégorie sont: $V$. paradoxa, $P$. biglobosa, A. occidentale, $C$. sinensis, $K$. senegalensis. La catégorie des taxa moyennement butinés est riche de trois (03), 10 et cinq (05) espèces respectivement pour les zones soudanienne, soudano-guinéenne et guinéenne ; et les plus fréquemment cités sont: $M$. indica, Lannea microcarpa, C. sinensis et Tectona grandis. $\mathrm{La}$ catégorie des espèces faiblement butinées comprend Azadirachta indica et Isoberlina doka. Une quatrième catégorie a été distinguée et prend en compte les taxa dont le degré de butinage n'a pas pu être clairement apprécié par les apiculteurs.

Le nombre moyen d'espèces citées par apiculteur a varié de 3,04 à 4,37 suivant la zone phytogéographique; de 3,03 à 4,8 au sein des communes; et de 3,06 à 4,36 au sein des groupes sociolinguistiques. La zone soudano-guinéenne est la plus diversifiée avec 34 espèces. A l'échelle des ethnies, ce sont les Bariba et apparentés puis les Yoruba et apparentés qui ont une forte connaissance des plantes mellifères (respectivement 24 et 22 espèces citées), contre 10 espèces chez les Peulh et apparentés. L'analyse de la variance a montré que le nombre moyen d'espèces citées par apiculteur varie en fonction de la zone phytogéographique, de la commune et de l'ethnie ( $\mathrm{p}<0,05)$.

Richesse taxonomique des plantes mellifères inductrices d'accumulation de miel

Les 31 espèces mellifères inductrices d'accumulation de miel citées par les apiculteurs appartiennent à 30 genres et 18 familles dont les plus diversifiées sont les Anarcadiaceae, les Bombacaceae, les 
Leguminosae-Cesalpinioideae,

les

Leguminosae-Papilionoideae et les Rutaceae (10\% chacune) (Tableau 2). En zones soudanienne et soudano-guinéenne, les espèces inductrices d'accumulation de miel les plus citées sont: P. biglobosa, V. paradoxa, A. occcidentale, M. indica, Ceiba pentandra. En zone guinéenne, $C$. sinensis, $K$. senegalensis et $E$. camadulensis ont les plus fortes fréquences de citation.

Le nombre moyen d'espèces inductrices d'accumulation de miel citées par apiculteur a varié de 1,51 à 2,26 suivant la zone phytogéographique; de 1,43 à 2,26 au sein des communes; et de 0,91 à 2,08 au sein des groupes sociolinguistiques. $\mathrm{La}$ zone soudano-guinéenne reste la plus diversifiée (21 espèces). A l'échelle des ethnies, ce sont les Bariba et apparentés puis les Fon et apparentés qui ont une forte connaissance des plantes inductrices d'accumulation de miel. L'analyse de la variance indique que le nombre moyen d'espèces nectarifères citées par apiculteur varie aussi bien en fonction de l'ethnie que de la zone phytogéographique.

\section{Espèces mellifères et traitement des maladies et/ou symptômes}

En médecine traditionnelle, 22 des 45 espèces mellifères citées sont utilisées (Tableau 3). Elles appartiennent à 15 familles dont les plus diversifiées sont les Leguminosae (36,36\%), les Anacardiaceae et les Rubiaceae (9,09\% chacune). Parmi les espèces mellifères thérapeutiques, les plus citées sont $V$. paradoxa $(28,6 \%), M$. indica $(12,8 \%), A$. occidentale et $P$. biglobosa (10,50\% chacune).

Cette flore mellifère médicinale est utilisée dans le traitement de 39 symptômes ou maladies. Le paludisme $(18,8 \%)$, les maux de ventre $(13,5 \%)$, la toux $(7,5 \%)$ et les maladies oculaires $(7,5 \%)$ sont les plus cités (Figure 3). En tenant compte de leur traitement, le paludisme implique à lui seul l'utilisation de $45,45 \%$ des espèces citées, ensuite viennent la toux $(27,27 \%)$, les hémorroïdes et les maux de ventre $(22,72 \%$ chacune), puis la carie dentaire, la diarrhée, les ictères, les infections et les ulcères (13,63\% chacune). L'analyse des indices du degré de consensus a montré que Acacia polyacantha ( $D C=0,80)$, Tamarindus indica $(D C=0,75)$ traitent préférentiellement le paludisme. Sarcocephalus latifolius $(D C=0,67)$ est majoritairement utilisée pour le traitement des maux de ventre, alors que $V$. paradoxa $(D C=0,50)$ est prisée aussi bien pour le traitement des maladies oculaires que pour celui des maux de ventre. $K$. senegalensis $(D C=0,50)$ est utilisée pour soigner les maux de ventre et les infections.

Tous les organes végétaux des espèces sont sollicités. Les écorces sont les plus utilisées $(50,4 \%)$, suivies des feuilles $(26,4 \%)$ et des racines $(13,5 \%)$. La décoction $(61,7 \%)$, la trituration sèche ou non $(17,3 \%)$ et la macération aqueuse ou alcoolique $(8,3 \%)$ sont les formes pharmaceutiques les plus utilisées. Dans 12\% des cas, les organes utilisés ne subissent aucun traitement préalable. $\mathrm{Au}$ regard des voies d'administration, 69,2\% des recettes sont utilisées en application interne. L'usage externe est cité dans $27,1 \%$ de cas, tandis que dans $3,8 \%$ de cas, l'administration des produits se fait à la fois par voie externe et par voie interne. Enfin, les espèces mellifères médicinales sont utilisées en association avec d'autres plantes ou ingrédients dans seulement $14,3 \%$ des recettes. 
Tableau 1 : Espèces visitées par les abeilles par zone phytogéographique.

\begin{tabular}{|c|c|c|c|c|c|c|c|c|}
\hline \multirow[t]{2}{*}{ Familles } & \multirow[t]{2}{*}{ Espèces } & \multirow{2}{*}{$\begin{array}{c}\text { Noms vernaculaires et } \\
\text { communs } \\
\end{array}$} & \multicolumn{3}{|c|}{ Degré de butinage } & \multicolumn{3}{|c|}{ Fréquence de citation $(\%)$} \\
\hline & & & $\mathrm{ZS}$ & $\overline{Z S G}$ & $\mathrm{ZG}$ & $\mathrm{ZS}$ & $\overline{\mathrm{ZSG}}$ & $\overline{Z G}$ \\
\hline \multirow{3}{*}{ Anacardiaceae } & Anacardium occidentale L. & Cajou $^{f r}$ & $F B$ & $F B$ & - & 9,30 & 65 & - \\
\hline & Lannea microcarpa Engl. \& K. Krause & Raisins sauvages $^{f r}$ & $M B$ & - & - & 11,63 & - & - \\
\hline & Mangifera indica $\mathrm{L}$ & Manguo $^{\text {an }}$ & $M B$ & $M B$ & $M B$ & 18,60 & 36,67 & 33,33 \\
\hline \multirow[t]{2}{*}{ Arecaceae } & Cocos nucifera L. & Coco $^{\text {fr }}$ & - & $M B$ & - & - & 1,67 & \\
\hline & Elaeis guineensis Jacq. & Palmier à huile $e^{f r}$ & - & $F B$ & $F B$ & - & 3,33 & 33,33 \\
\hline \multirow[t]{3}{*}{ Bombacaceae } & Adansonia digitata $\mathrm{L}$. & Baobab $^{f r}$, Kpassa $^{f o}$ & $I$ & $F B$ & - & - & 3,33 & - \\
\hline & Bombax costatum Pellegr. \& Vuillet & Hountin $^{\text {fo }}$, Bouabou $^{\text {wa }}$ & $F B$ & - & - & 2,33 & - & - \\
\hline & Ceiba pentandra $(\mathrm{L})$. & Guédégouxou ${ }^{\text {fo }}$ & $F B$ & $M B$ & - & 11,63 & 5 & - \\
\hline Caricaceae & Carica papaya $\mathrm{L}$. & Papayer $^{f r}$ & - & $M B$ & $M B$ & - & 6,67 & 3,33 \\
\hline Clusiacea & Pentadesma butyracea Sabine & Pentadesma $^{f r}$ & - & $F B$ & - & - & 1,67 & - \\
\hline \multirow[t]{2}{*}{ Combretaceae } & Anogeissus leiocarpa (DC.) Guill. \& Perr. & Goukankla $^{a n}$, Hlihoun $^{\text {fo }}$ & - & $F B$ & $F B$ & - & 1,67 & 3,33 \\
\hline & Combretum nigricans Lepr. ex Guill. et Perr. & Kouroundé ${ }^{w a}$ & $F B$ & - & - & 2,33 & - & - \\
\hline Composeae & Helianthus annuus L. & Tournesol $^{f r}$ & - & $I$ & $M B$ & - & - & 3,33 \\
\hline Euphorbiaceae & Flueggea virosa (Roxb. ex Willd.) Voigt. & Siansian $^{\text {ba }}$ & - & $F B$ & - & - & 3,33 & - \\
\hline \multirow{3}{*}{$\begin{array}{l}\text { Leguminosae- } \\
\text { Mimosoideae }\end{array}$} & Acacia polyacantha Willd. & Acacia $^{\text {fr }}$ & - & $F B$ & $F B$ & - & 6,67 & 20 \\
\hline & Albizia lebbeck (DC.) J. F. Macbr & Yodou $^{b a}$ & $M B$ & - & - & 4,65 & & - \\
\hline & Parkia biglobosa (Jacq.) R.Br. ex Benth & $\begin{array}{l}\text { Gourokou an, Néré }{ }^{f r}, \\
\text { Ogba }{ }^{\text {na }}\end{array}$ & $F B$ & $F B$ & - & 67,44 & 55 & - \\
\hline \multirow{6}{*}{$\begin{array}{l}\text { Leguminosae- } \\
\text { Cesalpinioideae }\end{array}$} & Afzelia africana $\mathrm{Sm}$. & Gouchélé $^{a n}$ & - & $F B$ & - & - & 1,67 & - \\
\hline & Caesalpinia pulcherrima (L.) Sw & Orgueil de chine ${ }^{f r}$ & - & $F B$ & - & - & 1,67 & - \\
\hline & Daniellia oliveri (Rolfe) Hutch. \& Dalziel & Iya $^{\text {na }}$, Gnambourou ${ }^{b a}$ & - & $F B$ & - & - & 3,33 & - \\
\hline & Detarium microcarpum Guill. \& Perr & Bessegolou $^{b a}$ & - & $F B$ & - & - & 11,67 & - \\
\hline & Isoberlina doka Craib \& Stapf & Gbagba $^{b a}$ & - & $F a B$ & - & - & 3,33 & - \\
\hline & Tamarindus indica $\mathrm{L}$. & $\underset{f r}{\text { Gouyemin }}{ }^{\text {an }}$, Tamarinier & $F B$ & $I$ & - & 4,65 & - & - \\
\hline \multirow{3}{*}{$\begin{array}{l}\text { Leguminosae- } \\
\text { Papilionoideae }\end{array}$} & Lonchocarpus sericeus (Poir.) Kunth & Ounssoubada ${ }^{f o}$ & - & - & $F B$ & - & - & 10 \\
\hline & Vigna unguiculata (L.) Walp & Niébé $e^{f r}$ & - & - & $I$ & - & - & - \\
\hline & Pterocarpus erinaceus Poir & Kosso $^{\text {wa, ba, }}$, Gokpala ${ }^{a n}$ & $I$ & $F B$ & - & - & 11,67 & - \\
\hline \multirow[t]{2}{*}{ Meliacea } & Azadirachta indica A. Juss & Neem $^{f r}$ & $F a B$ & $F B$ & - & 4,65 & 1,67 & - \\
\hline & Khaya grandifoliola CD.C & Faux cailcédgqgt $^{f r}$ & - & $F B$ & - & - & 1,67 & - \\
\hline
\end{tabular}




\begin{tabular}{|c|c|c|c|c|c|c|c|c|}
\hline & Khaya senegalensis (Desr.) A. Juss & Cailcédrat $^{\text {fr }}$ & $I$ & $M B$ & $F B$ & - & 3,33 & 50 \\
\hline & Melia azedarach $\mathrm{L}$. & Melia & - & $M B$ & $I$ & - & 5 & - \\
\hline Moraceae & Ficus platyphylla Del. & Kambo $^{\text {wa }}$ & $F B$ & - & - & 11,63 & - & - \\
\hline Myrtaceae & Eucalyptus camadulensis Dehn & Eucalyptus $^{f r}$ & $F B$ & - & $F B$ & 4,65 & - & 26,67 \\
\hline Olacaceae & Ximenia americana $\mathrm{L}$. & Minimou $^{\text {wa }}$, Gourokou ${ }^{\text {bar }}$ & - & - & - & - & 1,67 & - \\
\hline Pedaliaceae & Sesamum indicum L. & Sésame $^{f r}$ & - & $F B$ & - & - & 3,33 & - \\
\hline \multirow[t]{2}{*}{ Poaceae } & Zea mays L. & Mais $^{\text {fr }}$ & - & $M B$ & $M B$ & - & 3,33 & 6,67 \\
\hline & Sorghum bicolor $(\mathrm{L})$. & Sorgho ${ }^{f r}$ & - & $M B$ & - & - & 1,67 & - \\
\hline \multirow[t]{3}{*}{ Rubiaceae } & Crossopterix febrifuga (G. Don) Benth & Goupian $^{\text {an }}$ & - & $M B$ & - & - & 1,67 & - \\
\hline & Gardenia erubescens Staf \& Hutch & Bérébéréwa $^{\text {wa }}$ & $F B$ & - & - & 2,33 & - & - \\
\hline & Sarcocephalus latifolius (Sm.) E.A.Bruce & $\underset{b a}{\text { Komkombè }}{ }^{w a}$, Ganyérou & - & - & - & - & - & - \\
\hline \multirow[t]{3}{*}{ Rutaceae } & Citrus limon (L.) Burm. f. & Citronnier $^{f r}$ & - & $I$ & - & - & - & - \\
\hline & Citrus sinensis $\mathrm{L}$. & Oranger $^{f r}$ & - & $M B$ & $F B$ & - & 10 & 66,67 \\
\hline & Zanthoxylum zanthoxyloides (Lam.) & Zanthoxylum $^{\text {fr }}$ & - & $F B$ & - & - & 3,33 & - \\
\hline Sapotaceae & Vitellaria paradoxa C.F. Gaertn & Sakiribou ${ }^{\text {wa }}$ & $F B$ & $F B$ & - & 69,77 & 65 & - \\
\hline Tiliaceae & Corchorus olitorius L. & Yoiyoi $^{\text {na }}$ & - & $F B$ & - & - & 1,67 & - \\
\hline Verbenaceae & Tectona grandis L. f. & Teck $^{f r}$ & - & $I$ & $M B$ & - & - & 26,67 \\
\hline
\end{tabular}

ZS : Zone soudanienne; ZSG : Zone soudano-guinéenne; ZG : Zone guinéenne, an : ani ; ba : bariba ; fo : fon ; fr : français (nom usuel en langue française utilisé par les communautés rurales) ; na : nago ; wa : waama. FB : fortement butinée; MB : moyennement butinée; FaB : faiblement butinée; I : Fréquence de butinage indéterminé. 
Tableau 2 : Espèces mellifères inductrices d'accumulation de miel par zone phytogéographique.

\begin{tabular}{|c|c|c|c|c|c|}
\hline \multirow[t]{2}{*}{ Familles } & \multirow[t]{2}{*}{ Espèces } & \multirow{2}{*}{$\begin{array}{l}\text { Noms vernaculaires et } \\
\text { communs }\end{array}$} & \multicolumn{3}{|c|}{ Fréquence de citation $(\%)$} \\
\hline & & & $\mathrm{ZS}$ & $\overline{Z S G}$ & $\mathrm{ZG}$ \\
\hline \multirow{3}{*}{ Anacardiaceae } & Anacardium occidentale & Cajou $^{f r}$ & 4,65 & 40 & - \\
\hline & Lannea microcarpa & Raisins sauvages $^{f r}$ & 6,98 & - & - \\
\hline & Mangifera indica & Manguo $^{a n}$ & 4,65 & 23,33 & 23,33 \\
\hline Arecaceae & Elaeis guineensis & Palmier à huile ${ }^{f r}$ & - & 1,67 & 13,33 \\
\hline \multirow[t]{3}{*}{ Bombacaceae } & Adansonia digitata & Baobab $^{f r}$, Kpassa $^{f o}$ & - & 1,67 & 3,33 \\
\hline & Bombax costatum & Hountin $^{\text {fo }}$, Bouabou $^{\text {wa }}$ & - & - & 3,33 \\
\hline & Ceiba pentandra & Guédégouxou $^{\text {fo }}$ & 11,63 & - & - \\
\hline Caricaceae & Carica papaya & Papayer $^{f r}$ & - & 3,33 & - \\
\hline Combretaceae & Anogeissus leiocarpa & Goukankla $^{a n}$, Hlihoun $^{f o}$ & - & 1,67 & 3,33 \\
\hline Composeae & Helianthus annuus & Tournesol $^{f r}$ & - & 1,67 & - \\
\hline Euphorbiaceae & Flueggea virosa & Siansian $^{b a}$ & - & 1,67 & - \\
\hline \multirow{3}{*}{$\begin{array}{l}\text { Leguminosae- } \\
\text { Cesalpinioideae }\end{array}$} & Daniellia oliveri & Iya $^{n a}$, Gnambourou $^{b a}$ & - & 1,67 & - \\
\hline & Detarium microcarpum & Bessegolou $^{b a}$ & - & 8,33 & - \\
\hline & Tamarindus indica & Gouyemin $^{a n}$, Tamarinier $^{f r}$ & - & 1,67 & - \\
\hline \multirow{2}{*}{$\begin{array}{l}\text { Leguminosae- } \\
\text { Mimosoideae }\end{array}$} & Acacia polyacantha & Acacia $^{f r}$ & - & 1,67 & 13,33 \\
\hline & Parkia biglobosa & Gourokou $_{n a}^{a n,}$ Néré $^{f r,}$ Ogba & 58,14 & 31,67 & 3,33 \\
\hline \multirow{3}{*}{$\begin{array}{l}\text { Leguminosae- } \\
\text { Papilionoideae }\end{array}$} & Lonchocarpus sericeus & Ounssoubada $^{f o}$ & - & - & 10 \\
\hline & Pterocarpus erinaceus & Kosso $^{\text {wa, ba, }}$, Gokpala $^{a n}$ & 2,33 & 5 & - \\
\hline & Vigna unguiculata & Niébé $e^{f r}$ & 2,33 & - & - \\
\hline \multirow[t]{2}{*}{ Meliacea } & Khaya senegalensis & Cailcédrat $^{f r}$ & - & - & 43,33 \\
\hline & Melia azedarach & Melia & - & 1,67 & 3,33 \\
\hline Moraceae & Ficus platyphylla & Kambo $^{w a}$ & 4,65 & - & - \\
\hline Myrtaceae & $\begin{array}{l}\text { Eucalyptus } \\
\text { camadulensis }\end{array}$ & Eucalyptus $^{f r}$ & - & - & 40 \\
\hline Poaceae & Pennisetum glaucum & $M_{i l}^{f r}$ & 2,33 & - & - \\
\hline \multirow[t]{3}{*}{ Rutaceae } & Citrus limon & Citronnier $^{f r}$ & - & 1,67 & - \\
\hline & Citrus sinensis & Oranger $^{f r}$ & - & 3,33 & 63,33 \\
\hline & $\begin{array}{l}\text { Zanthoxylum } \\
\text { zanthoxyloides }\end{array}$ & Zanthoxylum $^{f r}$ & - & 3,33 & - \\
\hline Sapotaceae & Vitellaria paradoxa & Sakiribou $^{\text {wa }}$ & 53,49 & 46,67 & - \\
\hline Tiliaceae & Corchorus olitorius & Yoiyoi $^{n a}$ & - & 1,67 & - \\
\hline \multirow[t]{2}{*}{ Verbenaceae } & Tectona grandis & Teck $^{f r}$ & - & - & 3,33 \\
\hline & Vitex doniana & Vitex & - & 1,67 & - \\
\hline
\end{tabular}


Tableau 3 : Plantes inductrices d'accumulation de miel à usages médicinaux et leurs caractéristiques.

\begin{tabular}{|c|c|c|c|c|c|c|}
\hline $\begin{array}{l}\text { Noms } \\
\text { scientifiques }\end{array}$ & $\begin{array}{l}\text { FC } \\
(\%)\end{array}$ & $\begin{array}{ll}\text { Symptômes } & \text { ou } \\
\text { pathologies traités } & \\
\end{array}$ & $\begin{array}{l}\text { Organes } \\
\text { utilisées }\end{array}$ & $\begin{array}{l}\text { Mode de } \\
\text { préparation }\end{array}$ & $\begin{array}{l}\text { Voie } \\
\text { d'administration }\end{array}$ & DC \\
\hline \multirow{2}{*}{$\begin{array}{l}\text { Acacia } \\
\text { polyacantha }\end{array}$} & \multirow[t]{2}{*}{4,5} & Carie dentaire & Racines & Décoction & Boire & \multirow[t]{2}{*}{0,80} \\
\hline & & Paludisme & Feuilles & Décoction & Boire & \\
\hline $\begin{array}{l}\text { Adansonia } \\
\text { digitata }\end{array}$ & 0,8 & $\begin{array}{l}\text { Problèmes de faiblesse } \\
\text { des bébés }\end{array}$ & Ecorces & Décoction & Laver l'enfant & - \\
\hline $\begin{array}{l}\text { Afzelia } \\
\text { africana }\end{array}$ & 0,8 & Paludisme & Racines & Décoction & $\begin{array}{l}\text { Se laver avec } \\
\text { l'eau }\end{array}$ & - \\
\hline \multirow{9}{*}{$\begin{array}{l}\text { Anacardium } \\
\text { occidentale }\end{array}$} & \multirow{9}{*}{10,5} & Allergie & Ecorces & Décoction & Se laver avec & \multirow[t]{9}{*}{0,38} \\
\hline & & Carie dentaire & Ecorces, tiges & ------------- & Mâcher & \\
\hline & & Troubles acnéiques & Ecorces & Décoction & Se laver avec & \\
\hline & & Hémorroïde & Ecorces & Décoction & Boire & \\
\hline & & Paludisme & Feuilles jeunes & Décoction & $\begin{array}{l}\text { Boire et recouvrir } \\
\text { le corps }\end{array}$ & \\
\hline & & Plaies buccales & Ecorces & Décoction & Gargarisme & \\
\hline & & $\begin{array}{l}\text { Problèmes de tension } \\
\text { artérielle }\end{array}$ & Ecorces & Décoction & Boire & \\
\hline & & Toux & $\begin{array}{l}\text { Feuilles } \\
\text { sèches/Ecorce/T } \\
\text { ige }\end{array}$ & $\begin{array}{l}\text { Décoction / } \\
\text { Aucun }\end{array}$ & Boire/mâcher & \\
\hline & & Ulcère & Ecorces & Décoction & Boire & \\
\hline $\begin{array}{l}\text { Caesalpinia } \\
\text { pulcherrima }\end{array}$ & 0,8 & Toux & Feuilles & Décoction & Boire & - \\
\hline \multirow[t]{4}{*}{$\begin{array}{l}\text { Carica } \\
\text { papaya }\end{array}$} & \multirow[t]{4}{*}{5,3} & $\begin{array}{l}\text { Difficultés lors de } \\
\text { l'accouchement }\end{array}$ & Racines & --------------- & Mâcher & \multirow[t]{4}{*}{0,50} \\
\hline & & Ictère & Fruits non mûrs & $\begin{array}{l}\text { Macération } \\
\text { (eau) }\end{array}$ & Boire & \\
\hline & & Paludisme & Feuilles jaunes & Décoction & Boire & \\
\hline & & $\begin{array}{l}\text { Problèmes de piqure de } \\
\text { scorpion }\end{array}$ & Racines & $\begin{array}{l}\text { Trituration } \\
\text { (après } \\
\text { chauffage) }\end{array}$ & Application locale & \\
\hline Citrus limon & 0,8 & Constipation & Fruits & Presser & Boire & - \\
\hline $\begin{array}{l}\text { Crossopterix } \\
\text { febrifuga }\end{array}$ & 0,8 & Diarrhée & Ecorces & Décoction & Boire & - \\
\hline $\begin{array}{l}\text { Detarium } \\
\text { microcarpum }\end{array}$ & 0,8 & Maladies cardiaques & Ecorces & Trituration & Laper & - \\
\hline \multirow{2}{*}{$\begin{array}{l}\text { Eucalyptus } \\
\text { camadulensis }\end{array}$} & \multirow[t]{2}{*}{1,5} & Rhume & Feuilles & Décoction & Boire & \multirow[t]{2}{*}{0} \\
\hline & & Toux & Feuilles & Décoction & Boire & \\
\hline $\begin{array}{l}\text { Ficus } \\
\text { platyphylla }\end{array}$ & 0,8 & Paludisme & Feuilles & Décoction & Recouvrir le corps & - \\
\hline $\begin{array}{l}\text { Flueggea } \\
\text { virosa }\end{array}$ & 0,8 & Problème d'entorse & Racines & Trituration & Application locale & - \\
\hline $\begin{array}{l}\text { Helianthus } \\
\text { annuus }\end{array}$ & 0,8 & Toux & Feuilles & Décoction & Boire & - \\
\hline \multirow{2}{*}{$\begin{array}{l}\text { Isoberlina } \\
\text { doka }\end{array}$} & \multirow[t]{2}{*}{1,5} & Dysménorrhées & Ecorces & Décoction & Boire & \multirow[t]{2}{*}{0} \\
\hline & & Infections & Feuilles & Décoction & Boire & \\
\hline \multirow[t]{3}{*}{$\begin{array}{l}\text { Khaya } \\
\text { senegalensis }\end{array}$} & \multirow[t]{3}{*}{3,8} & Infections & Ecorces & $\begin{array}{l}\text { Macération } \\
\text { (alcool)/Dé } \\
\text { coction }\end{array}$ & Boire & \multirow[t]{3}{*}{0,50} \\
\hline & & Maux de ventre & Ecorces & $\begin{array}{l}\text { Trituration/ } \\
\text { Décoction }\end{array}$ & Laper ou boire & \\
\hline & & Dysménorrhées & Ecorces & Trituration & Prendre avec du & \\
\hline
\end{tabular}




\begin{tabular}{|c|c|c|c|c|c|c|}
\hline & & & & & citron & \\
\hline \multirow{13}{*}{$\begin{array}{l}\text { Mangifera } \\
\text { indica }\end{array}$} & \multirow[t]{13}{*}{12,8} & Carie dentaire & Ecorces & Décoction & Boire & \multirow[t]{13}{*}{0,38} \\
\hline & & Fièvre & Ecorces & Trituration & $\begin{array}{l}\text { Application } \\
\text { générale }\end{array}$ & \\
\hline & & Hémorroïde & Ecorces & Décoction & $\begin{array}{l}\text { Application locale } \\
\text { (vapeur) }\end{array}$ & \\
\hline & & Ictère & Racines & Décoction & Boire & \\
\hline & & Maux des yeux & $\begin{array}{l}\text { Ecorces } \\
\text { Feuilles }\end{array}$ & $\begin{array}{l}\text { Macération } \\
\text { (eau) }\end{array}$ & Laver le visage & \\
\hline & & Maux de ventre & Ecorces & $\begin{array}{l}\text { Trituration/ } \\
\text { Décoction }\end{array}$ & Boire & \\
\hline & & Paludisme & Ecorces & Décoction & $\begin{array}{l}\text { Boire et recouvrir } \\
\text { le corps }\end{array}$ & \\
\hline & & Contusions & Ecorces & Décoction & $\begin{array}{l}\text { Essuyer avec la } \\
\text { solution }\end{array}$ & \\
\hline & & Plaies buccales & Ecorces & Décoction & Gargarisme & \\
\hline & & Ulcère & Ecorces & Décoction & Boire & \\
\hline & & Troubles helminthiques & Ecorces & Décoction & Boire & \\
\hline & & Maux de ventre & Racines & $\begin{array}{l}\text { Décoction/ } \\
\text { aucun } \\
\text { traitement }\end{array}$ & Boire/Mâcher & \\
\hline & & Ictère & Racines & Décoction & Boire & \\
\hline \multirow[t]{10}{*}{$\begin{array}{l}\text { Parkia } \\
\text { biglobosa }\end{array}$} & \multirow[t]{10}{*}{10,5} & Troubles de l'appétit & Ecorces & Trituration & $\begin{array}{l}\text { Prendre avec de } \\
\text { l'eau }\end{array}$ & \multirow[t]{10}{*}{0,31} \\
\hline & & Dentition & Feuilles & Décoction & $\begin{array}{l}\text { Boire/Laver } \\
\text { l'enfant }\end{array}$ & \\
\hline & & Dysenterie & Ecorces & Trituration & Laper & \\
\hline & & Troubles acnéiques & Feuilles & Trituration & Application locale & \\
\hline & & Hémorroïde & Ecorces & Trituration & Laper & \\
\hline & & Hernie & Tiges & -------------- & Mâcher & \\
\hline & & Maux de ventre & Ecorces & Trituration & $\begin{array}{l}\text { Prendre avec de } \\
\text { l'eau }\end{array}$ & \\
\hline & & Paludisme & Feuilles & Trituration & $\begin{array}{l}\text { Boire avec un } \\
\text { liquide }\end{array}$ & \\
\hline & & Toux & Ecorces/Fruits & Décoction & Boire & \\
\hline & & Varicelle & Feuilles/Ecorces & $\begin{array}{l}\text { Trituration/ } \\
\text { Décoction }\end{array}$ & $\begin{array}{l}\text { Application } \\
\text { locale/Boire }\end{array}$ & \\
\hline $\begin{array}{l}\text { Pentadesma } \\
\text { butyracea }\end{array}$ & 0,8 & Asthme & Racines & $\begin{array}{l}\text { Macération } \\
\text { (eau) }\end{array}$ & Boire & - \\
\hline \multirow{5}{*}{$\begin{array}{l}\text { Pterocarpus } \\
\text { erinaceus }\end{array}$} & \multirow[t]{5}{*}{4,5} & Anémie & Ecorces & Décoction & Boire & \multirow[t]{5}{*}{0,20} \\
\hline & & Diarrhée & Ecorces & Décoction & Boire & \\
\hline & & Teigne & Sève & $\begin{array}{l}-------------- \\
-----\end{array}$ & Application locale & \\
\hline & & Hémorroïde & Ecorces & $\begin{array}{l}\text { Macération } \\
\text { (eau) }\end{array}$ & Boire & \\
\hline & & Paludisme & Racines & Décoction & Boire & \\
\hline \multirow[t]{2}{*}{$\begin{array}{l}\text { Sarcocephalu } \\
\text { s latifolius }\end{array}$} & \multirow[t]{2}{*}{5,3} & Paludisme & Racines & $\begin{array}{l}\text { Macération } \\
(\text { eau)/ } \\
\text { Décoction }\end{array}$ & $\begin{array}{l}\text { Boire/ Se laver } \\
\text { avec la solution }\end{array}$ & \multirow[t]{2}{*}{0,67} \\
\hline & & Maux de ventre & Racines & $\begin{array}{l}\text { Décoction/ } \\
\text { aucun } \\
\text { traitement }\end{array}$ & Boire/Mâcher & \\
\hline
\end{tabular}




\begin{tabular}{|c|c|c|c|c|c|c|}
\hline & & Ictère & Racines & Décoction & Boire & \\
\hline \multirow[t]{2}{*}{$\begin{array}{l}\text { Tamarindus } \\
\text { indica }\end{array}$} & \multirow[t]{2}{*}{3,8} & Paludisme & Fruits & $\begin{array}{l}\text { Macération } \\
(\mathrm{eau}) / \text { Déco } \\
\text { ction }\end{array}$ & Boire & \multirow[t]{2}{*}{0,75} \\
\hline & & Dysenterie & Feuilles & Décoction & Boire & \\
\hline \multirow{20}{*}{$\begin{array}{l}\text { Vitellaria } \\
\text { paradoxa }\end{array}$} & \multirow[t]{20}{*}{28,6} & & & & & \multirow[t]{20}{*}{0,51} \\
\hline & & Allergie & Fleurs & Décoction & Humer & \\
\hline & & Angine & Ecorces & Décoction & Boire & \\
\hline & & Diarrhée & Ecorces & Décoction & Boire & \\
\hline & & Dysenterie & Ecorces & $\begin{array}{l}\text { Décoction / } \\
\text { Trituration }\end{array}$ & Boire/ Laper & \\
\hline & & Problème d'entorse & Feuilles & Trituration & Application locale & \\
\hline & & $\begin{array}{l}\text { Problèmes de faiblesse } \\
\text { des bébés }\end{array}$ & Feuilles & Décoction & Laver l'enfant & \\
\hline & & Fatigue & Ecorces & Trituration & $\begin{array}{l}\text { Prendre avec de } \\
\text { l'eau }\end{array}$ & \\
\hline & & Fièvre & Feuilles & Décoction & Boire & \\
\hline & & Hémorroïde & $\begin{array}{l}\text { Racines } \\
\text { pelées /Ecorces }\end{array}$ & $\begin{array}{l}\text { Trituration/ } \\
\text { Décoction }\end{array}$ & $\begin{array}{l}\text { Application } \\
\text { locale/boire }\end{array}$ & \\
\hline & & Infections & Ecorces & $\begin{array}{l}\text { Macération } \\
\text { (eau) }\end{array}$ & Boire & \\
\hline & & $\begin{array}{l}\text { Problèmes } \quad \mathrm{de} \\
\text { démangeaison }\end{array}$ & Ecorces & Décoction & Application locale & \\
\hline & & Maux de tête & Ecorces & Décoction & Boire & \\
\hline & & Maux d'yeux & $\begin{array}{l}\text { Ecorces /Racine } \\
\text { s/ Feuilles }\end{array}$ & $\begin{array}{l}\text { Macération } \\
\text { (eau)/ } \\
\text { Trituration/ } \\
\text { Décoction }\end{array}$ & $\begin{array}{l}\text { Laver } \\
\text { visage/Boire }\end{array}$ & \\
\hline & & Maux de ventre & $\begin{array}{l}\text { Ecorces/Feuilles } \\
\text { /Tiges }\end{array}$ & $\begin{array}{l}\text { Décoction / } \\
\text { Trituration/ } \\
\text { Aucun }\end{array}$ & $\begin{array}{l}\text { Boire/Laper/Mâch } \\
\text { er }\end{array}$ & \\
\hline & & $\begin{array}{l}\text { Problèmes de morsure de } \\
\text { serpent }\end{array}$ & Ecorces & Trituration & Application locale & \\
\hline & & Contusions & Sève & ---------- & Application locale & \\
\hline & & Toux & Ecorces & Décoction & Boire & \\
\hline & & Ulcère & Ecorces & Décoction & Boire & \\
\hline & & Vertige & Ecorces & Décoction & $\begin{array}{l}\text { Boire et se laver } \\
\text { le visage }\end{array}$ & \\
\hline
\end{tabular}

FC : Fréquence de citation ; DC : Degré de consensus. 


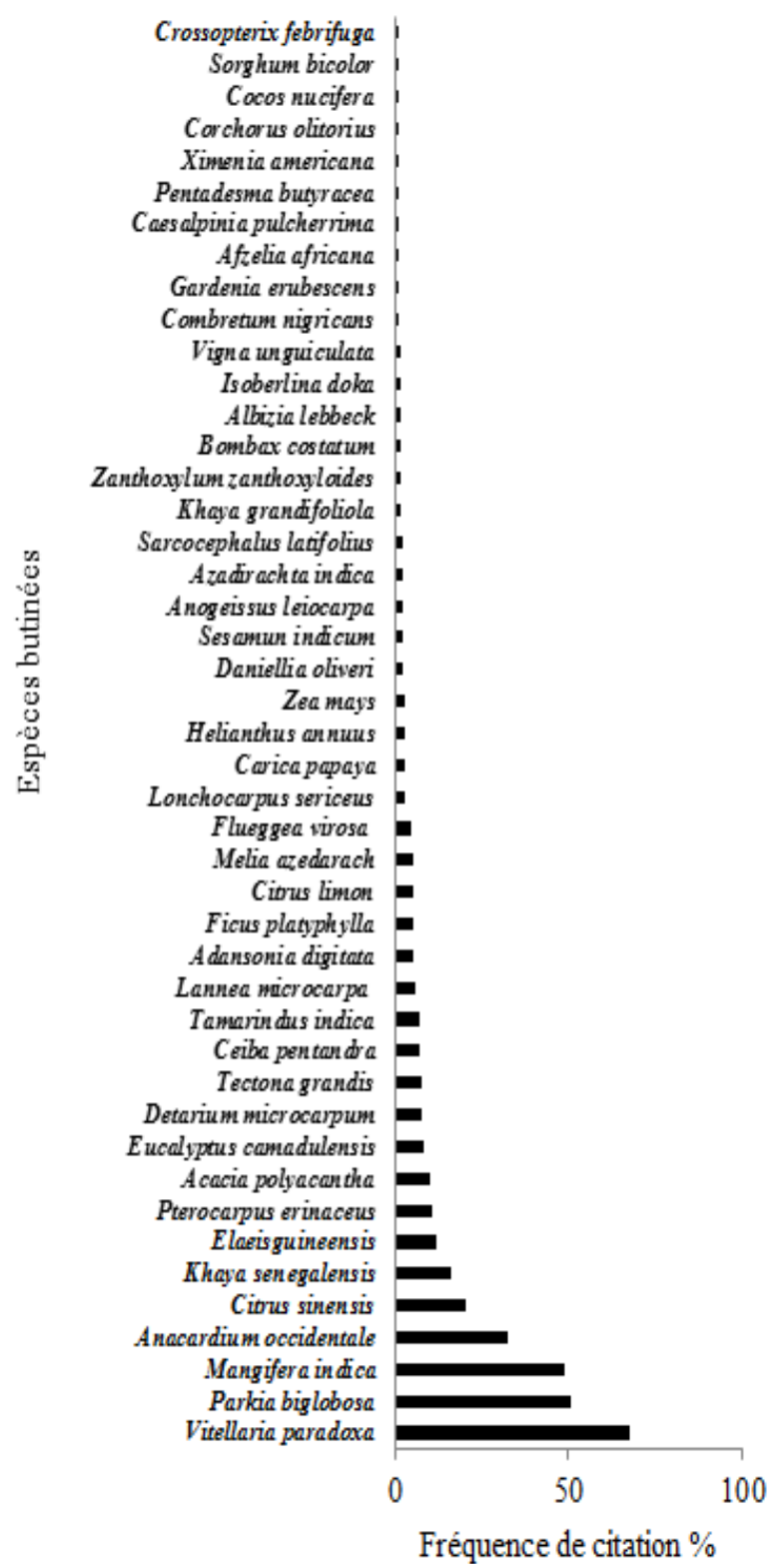

Figure 2 : Fréquence de citation des espèces visitées par les abeilles.

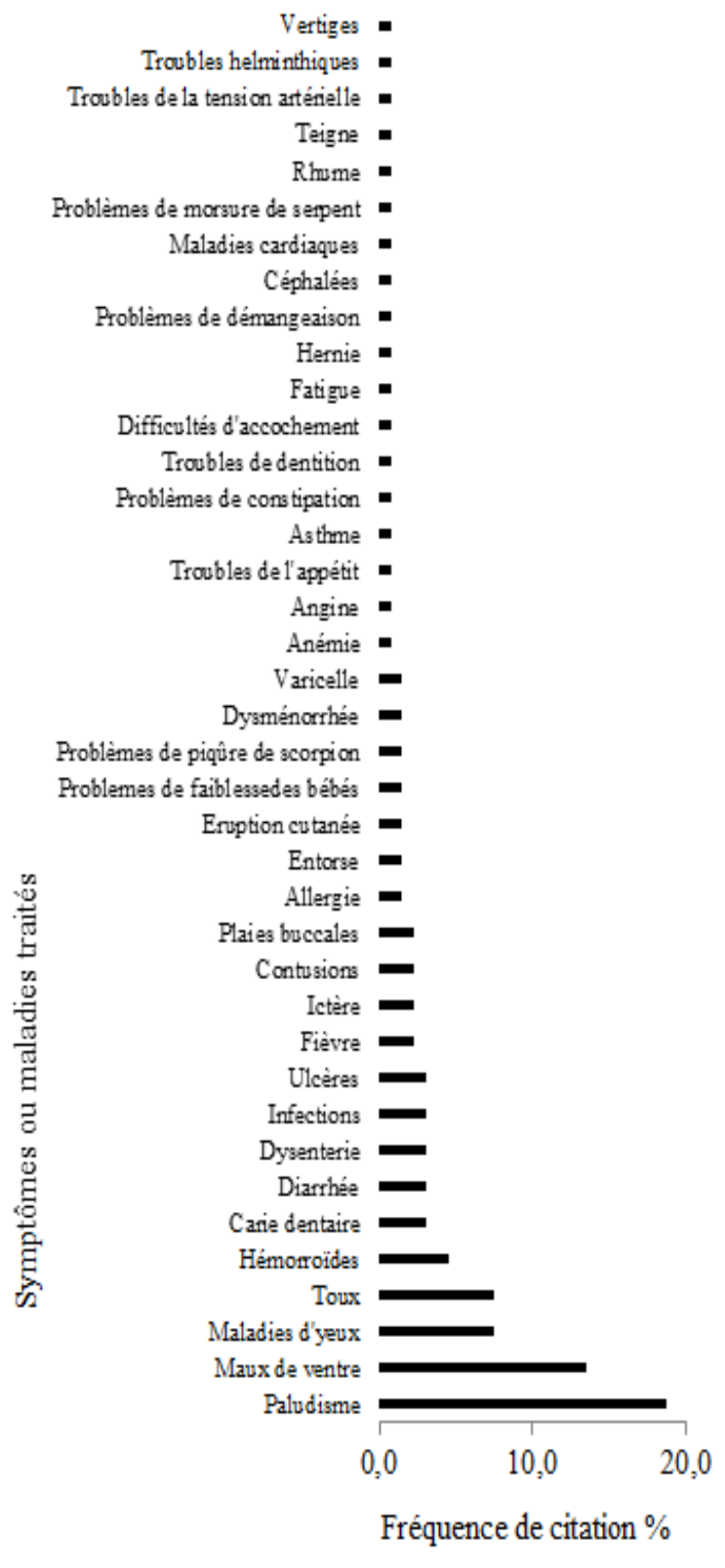

Figure 3 : Fréquence de citation des 39 symptômes et affections. 


\section{DISCUSSION}

Sur le plan de la diversité des familles, la citation prédominante des Leguminosae par les populations, remarquée également par Amakpe et al. (2015), n'est pas surprenante. Cette famille constitue, en effet, une caractéristique générale des formations végétales naturelles des zones soudanoguinéenne et soudanienne, deux des trois zones de notre milieu d'étude (Nombré, 2003). La forte citation des espèces de la sous famille des Mimosoideae dans les zones soudanienne et soudano-guinéenne est justifiée par les affirmations de Yédomonhan et al. (2009), qui stipulent que cette sous famille renferme des espèces à haute valeur mellifère qui constituent un atout floristique intéressant pour la production du miel. En outre, dans les zones soudanienne et soudanoguinéenne, $V$. paradoxa reste l'espèce mellifère la plus citée par les apiculteurs. Ce caractère hautement mellifique de $V$. paradoxa a déjà été observé par Amakpè et al. (2015), qui affirment qu'elle est l'espèce mellifère endémique la plus importante au Nord du Bénin. Cette importance est en outre due, selon ces auteurs, à sa haute valeur économique (production de beurre de karité). La citation des plantes mellifères a suivi un gradient décroissant de la zone soudanienne à la zone guinéenne en passant par la soudanoguinéenne. Ainsi, les zones soudanienne et soudano-guinéenne restent les plus riches en espèces mellifères. Une telle observation a déjà été faite par Yédomonhan et al. (2009) qui affirment que la zone soudano-guinéenne regorge plus d'espèces que la zone guinéenne. Sur les 45 espèces mellifères citées par les apiculteurs, 42 sont effectivement recensées comme telles dans la littérature selon les travaux de Telleria (1988) en Argentine, Dongock et al. (2004) au Cameroun, Yédomonhan (2009), Yédomonhan et al. (2009) et Amakpè et al. (2015) au Bénin, Saha (2006) au Bengladesh, Nombré et al. (2009) et FAO (2012) au Burkina-Faso, Koudegan et al. (2012) au Togo, Iritié et al. (2014) en Côte d'Ivoire: soit un taux de conformité de
93,33\% entre les connaissances des apiculteurs et les données de la littérature. Seules trois espèces (Pentadesma butyraceae, Ficus platyphylla et $K$. grandifolia) n'ont pas pu être identifiées dans la littérature comme mellifères. Toutefois d'autres espèces du genre Ficus (Ficus elastica, Ficus capensis, Ficus leprieuri) ont été enregistrées comme espèces mixtes (Iritié et al., 2014). Sur les 31 espèces citées comme inductrices d'accumulation du miel, 29 espèces soit $93,54 \%$ sont réellement nectarifères d'après les travaux des auteurs suscités; et deux pollinifères. Tous ces résultats permettent de dire qu'au Bénin, la connaissance des apiculteurs en matière d'espèces mellifères est satisfaisante. Ceci reste un grand atout pour l'essor de la filière apicole.

Le paludisme qui vient en tête de liste dans les pathologies citées a été également enregistré par Adomou et al. (2012) puis Béné et al. (2016) comme l'affection la plus traitée par la médecine traditionnelle en Afrique. Cette forte mise en cause du paludisme est sûrement liée au fait qu'il y est une maladie endémique qui s'accompagne de multiples symptômes (fièvre, vomissements, céphalées et fatigue). Quant aux spécificités thérapeutiques, l'espèce mellifère la plus citée, $V$. paradoxa, a été enregistrée par Adomou et al. (2012) pour le traitement des maux de ventre comme il a été le cas dans cette étude. Dans la littérature, elle a aussi été enregistrée comme un emménagogue (Koko et al., 2011). Des vertus antipaludiques ont, par ailleurs, été attribuées aux espèces du genre Acacia, à Carica papaya et à Tamarindus indica (Sereme et al., 2008 ; Lakouéténé et al., 2009 ; Farzana et al., 2014). Ces résultats corroborent les vertus thérapeutiques recensées par cette étude pour ces différentes espèces. L'utilisation de K. senegalensis pour le traitement des maux de ventre et des infections (Adomou et al., 2012) confirme également les résultats de cette étude. Par ailleurs, une activité hépato-protective lui est aussi attribuée (Ali et al., 2011), et les extraits de ses racines seraient actifs contre la syphilis. 
L'utilisation de Adansonia digitata et de A. occidentale dans le traitement respectif du retard de croissance chez l'enfant et de l'hypertension artérielle a été également mise en évidence par Zerbo et al. (2007) puis Sereme et al. (2008).

La décoction enregistrée comme la principale forme pharmaceutique avec une prédominance de l'administration des recettes par voie orale a été rapportée par de nombreux chercheurs (Zerbo et al., 2007 ; Lakouéténé et al., 2009; Bakwaye et al., 2013 ; Ambe et al., 2015 ; Béné et al., 2016 ; Tsobou et al., 2016). Elle est souvent suivie de la macération aqueuse ou de l'infusion. Selon Salhi et al. (2010), la décoction permet en effet, de recueillir le plus de principes actifs et atténue ou annule l'effet toxique de certaines recettes. Contrairement aux résultats de cette étude qui ont montré une grande utilisation des écorces dans les recettes, nombreux sont les auteurs suscités qui ont plutôt noté une grande utilisation des feuilles. Cette différence pourrait s'expliquer par le fait que l'utilisation d'un organe est conjointement fonction de la pathologie à traiter et de l'espèce mise en cause. Toutefois, l'utilisation majoritaire des écorces observée est conforme aux conclusions de Schweitzer et al. (2013) au Burkina Faso. Enfin, la prédominance des recettes monospécifiques $(85,7 \%)$ observée est confirmée par les travaux de Ndjouondo et al. (2015) puis de Béné et al. (2016). Cette prépondérance est à l'avantage des patients puisque les associations de plantes mal assorties sont parfois dangereuses.

\section{Conclusion}

Le Bénin, en raison de sa diversité floristique, faunistique et paysagère est doté d'un potentiel apicole important. Cette étude a permis d'apprécier la diversité et la valeur socio-culturelle des espèces mellifères dans les trois zones phytogéographique du Bénin.

Les résultats rendent compte de l'intérêt des apiculteurs béninois pour les relations qui existent entre les abeilles et les plantes. Cet intérêt reste un atout exploitable pour le développement de l'activité apicole dont les potentialités sont encore sousexploitées. Les espèces mellifères sont également fortement impliquées en médecine traditionnelle. La connaissance des vertus de ces plantes, vertus dont certaines ont déjà été documentées dans la littérature, demeure le point de départ de la compréhension des vertus thérapeutiques des produits de la ruche.

\section{CONFLIT D'INTERETS}

Les auteurs déclarent qu'ils n'ont aucun conflit d'intérêts.

\section{CONTRIBUTIONS DES AUTEURS}

SCL a assuré la collecte des données de cette étude, les a traitées et a rédigé l'article. HY, FPT, AJD et AA ont suivi le traitement des données et ont participé à la lecture de cet article.

\section{REMERCIEMENTS}

Les auteurs de cet article tiennent à remercier les apiculteurs de Bassila, de N'dali, de Tanguiéta, de Kandi et de Zogbodomey pour leur assistance et leur ouverture.

\section{REFERENCES}

Adomou AC, Yédomonhan H, Djossa B, Legba SI, Oumorou M, Akoegninou A. 2012. Etude Ethnobotanique des plantes médicinales vendues dans le marché d'Abomey-Calavi au Bénin. Int. J. Biol. Chem. Sci., 6(2): 745-772. DOI: http://dx.doi.org/10.4314/ijbcs.v6i2.18

Akoègninou A, Van der Burg WJ, Van der Maesen LJG. 2006. Flore Analytique du Bénin. Backhuys Publishers; p. 1063.

Ali SAM, El Badwi SMA, Idris TM, Osman KM. 2011. Hepato-protective activity of aqueous extract of Khaya senegalensis bark in rats. J. Med. Plants Res., 5(24): 5863-5866.

Amakpè F, Akouehou GS, de Graaf DC, Sinsin B. 2015. Determination of the silvo-melliferous regions of Benin: a nationwide categorisation of the land based on melliferous plants suitable for 
timber production. J. Agr. Rural Develop. Trop. Subtrop., 116(2): 143156. DOI: http://nbnresolving.de/urn:nbn:de:hebis:342015061048473

Ambe ASA, Ouattara D, Tiebre MS, Vroh BTA, Zirihi GN, N'Guessan KE. 2015. Diversité des plantes médicinales utilisées dans le traitement traditionnel de la diarrhée sur les marchés d'Abidjan (Côte d'Ivoire). J. Anim. Plant Sci., 26(2): 4081-4096. http://www.m.elewa.org/JAPS

Atibita ENO, Fohouo FNT, Djieto-Lordon C. 2015. Activité de butinage et de pollinisation de Apis mellifera adansonii Latreille (Hymenoptera : Apidae) sur les fleurs de Oxali barrelieri (Oxalidaceae) à Yaoundé (Cameroun). Entomol. Faun., 68 : 101-108.

Bakwaye FN, Termote C, Kibungu K, Van Damme P. 2013. Identification et importance locale des plantes médicinales utilisées dans la région de Mbanza-Ngungu, République démocratique du Congo. Bois For Trop, 316(2): p.16. DOI : https://doi.org/10.19182/bft2013.316.a20 531

Béné K, Camara D, Fofie NBY, Kanga Y, Yapi AB, Yapo YC, Ambe SA, Zirihi GN. 2016. Étude ethnobotanique des plantes médicinales utilisées dans le Département de Transua, District du Zanzan (Côte d'Ivoire). J. Anim. Plant Sci., 27(2): 4230-4250. http://www.m.elewa.org/JAPS

Dongock DN, Foko J, Pinta JY, Ngouo LV, Tchoumboue J, Zango P. 2004. Inventaire et identification des plantes mellifères de la zone soudano-guinéenne d'altitude de l'ouest Cameroun. Tropicultura, 22(3) : 139-145.

Dro B, Soro D, Koné MW, Bakayoko A, Kamanzi K. 2013. Evaluation de l'abondance de plantes médicinales utilisées en médecine traditionnelle dans le Nord de la Côte d'Ivoire. J. Anim.
Plant Sci., 17(3): 2631-2646. http://www.m.elewa.org/JAPS

Farzana MUZN, Tharique IAl, Arshiya S. 2014. A review of ethnomedicine, phytochemical and pharmacological activities of Acacia nilotica (Linn) willd. J. Pharmacogn Phytochem, 3: 84-90.

FAO (Food and Agriculture Organization). 2012. Produits forestiers non ligneux. Rapport national du Burkina Faso, p.14.

Fosto KPR, Meutchieye F, Andriamanalina SI, Youbissi A, Tchoumboué J, Pinta JY, Zango P. 2014. Caractéristiques socioéconomiques et techniques de l'apiculture dans les Départements de Bamboutos, Mifi et Menoua (Région de l'Ouest-Cameroun). LRRD, 26(12): p. 20.

Iritie BM, Eboua NW, Paraïso AA, Fantodji A, Gbomene LL. 2014. Identification des plantes mellifères de la zone agroforestière de l'école supérieure agronomique de Yamoussoukro (Côte d'Ivoire). Eur. Sci. J., 10(30): p. 15. DOI :

http://dx.doi.org/10.19044/esj.2014.v10n $30 \mathrm{p} \% 25 \mathrm{p}$

Koko IKED, Djego J, Gbenou J, HounzangbeAdote SM, Sinsin B. 2011. Etude phytochimique des principales plantes galactogènes et emménagogues utilisées dans les terroirs riverains de la zone cynégétique de la Pendjari. Int. J. Biol. Chem. Sci., 5: 618-633. DOI: http://dx.doi.org/10.4314/ijbcs.v5i2.7212 7

Koudegnan KM, Edorh TM, Guelly AK, Batawilla K, Akpagana K. 2012. Inventaire des taxons polliniques des miels de la zone guinéenne du Togo : cas des zones écofloristiques IV ET V. Eur. Sci. J., 8(26): p. 14. DOI : http://dx.doi.org/10.19044/esj.2012.v8n2 $6 \mathrm{p} \% 25 \mathrm{p}$

Lakouéténé DPB, Ndolngar G, Berké B, Moyen JM, Kosh Komba E, Zinga I, Silla S, Millogo-Rasolodimby J, Vincendeau P, Syssa-Magalé JL, 
Nacoulma-Ouedraogo OG, Laganier R, Badoc A, Chèze C. 2009. Enquête ethnobotanique des plantes utilisées dans le traitement du paludisme à Bangui. Bull. Soc. Pharm. Bordeaux, 148 : 123138.

Ndjouondo GP, Ngene JP, Ngoule CC, Kidik Pouka MC, Ndjib RC, Dibong SD, Mpondo Mpondo E. 2015. Inventaire et caractérisation des plantes médicinales des sous bassins versants Kambo et Longmayagui (Douala, Cameroun). $J$. Anim. Plant Sci., 25(3): 3898-3916. http://www.m.elewa.org/JAPS

Nombré I. 2003. Etudes des potentialités mellifères de deux zones du Burkina Faso : Garango (province du Bouglou) et Nazinga (province du Nahouri). Thèse de Doctorat, Université de Ouagadougou, Burkina-Faso, p. 156.

Nombré I, Schweitzer P, Sawadogo M, Boussim JI, Millogo-Rasolodimby J. 2009. Assessment of melliferous plant potentialities in Burkina Faso. Afr. $J$. Ecol., 47: 622-629. DOI : https://doi.org/10.1111/j.13652028.2009.01034.x

Saha JC. 2006. L'apiculture pour le développement rural - son potentiel- et l'apiculture contre la pauvreté - une perspective de Bangladesh. http://www.apimondiafoundation.org/fou ndation/files/018f, (Consulté le 23/06/2016).

Salhi S, Fadli M, Zidane L, Douira A. 2010. Etudes floristique et ethnobotanique des plantes médicinales de la ville de Kénitra (Maroc). Lazaroa, 31: 133-146. DOI:10.5209/rev_LAZA.2010.v31.9
Schweitzer P, Nombré I, Kwamé A, Joseph BI. 2013. Plants used in traditional beekeeping in Burkina Faso. OJE, 3(5): 354-358.

DOI: http://dx.doi.org/10.4236/oje.2013.35040 Sereme A, Millogo-Rasolodimby J, Guinko S, Nacro M. 2008. Propriétés thérapeutiques des plantes à tanins du Burkina Faso. Pharm. Méd. Trad. Afr., 15 : 41-49.

Telleria MC. 1988. Analyse pollinique des miels du nord-ouest de la province de Buenos Aires (République argentine). Apidologie, 19(3): 275-290.

Tsobou R, Apongmetsem PM, Van Damme P. 2016. Medicinal Plants Used for Treating Reproductive Health Care Problems in Cameroon, Central Africa. Econ. Bot., $\quad \mathbf{7 0}(2) \quad 145-159$. DOI : $10.1007 / \mathrm{s} 12231-016-9344-0$

Yédomonhan H. 2009. Plantes mellifères et potentialités de production de miel en zones guinéenne et soudano-guinéenne au Bénin. Thèse de doctorat, Université d'Abomey-Calavi, Bénin, p. 294.

Yédomonhan $\mathrm{H}$, Tossou GM, Akoègninou $\mathrm{A}$, Demenou BB, Traore D. 2009. Diversité des plantes mellifères de la zone soudano-guinéenne : cas de l'arrondissement de Manigri (CentreOuest du Bénin). Int. J. Biol. Chem. Sci., 3(2): $\quad 355-366 . \quad$ DOI: http://dx.doi.org/10.4314/ijbcs.v3i2.4451 4

Zerbo P, Millogo-Rasolodimby J, NacoulmaOuedraogo OG, Van Damme P. 2007. Contribution à la connaissance des plantes médicinales utilisées dans les soins infantiles en pays San, au Burkina Faso. Int. J. Biol. Chem. Sci., 1(3) : 262. 